\title{
Handling stress may confound murine gut microbiota studies
}

\author{
Cary R. Allen-Blevins ${ }^{1}$ ， Xiaomeng You $^{2}$ ， Katie Hinde ${ }^{3,4}$ ， David A Sela ${ }^{\text {Corresp. 2, 5, } 6}$ \\ 1 Department of Human Evolutionary Biology, Harvard University, Cambridge, MA, United States \\ 2 Dept. of Food Science, University of Massachusetts, Amherst, MA, USA \\ 3 Center for Evolution and Medicine, Arizona State University, Tempe, AZ, United States \\ 4 School of Human Evolution and Social Change, Arizona State University, Tempe, AZ, United States \\ 5 Dept. of Microbiology, University of Massachusetts, Amherst, MA, USA \\ ${ }^{6}$ Center for Microbiome Research, University of Massachusetts Medical School, Worcester, MA, United States \\ Corresponding Author: David A Sela \\ Email address: davidsela@umass.edu
}

Background. Accumulating evidence indicates interactions between human milk composition, particularly sugars (human milk oligosaccharides or HMO), the gut microbiota of human infants, and behavioral effects. Some HMO secreted in human milk is unable to be endogenously digested by the human infant but are able to be metabolized by certain species of gut microbiota, including Bifidobacterium longum subsp. infantis (B. infantis), a species sensitive to host stress (Bailey \&Coe 2004). Exposure to gut bacteria like $B$. infantis during critical neurodevelopment windows in early life appears to have behavioral consequences; however, environmental, physical, and social stress during this period can also have behavioral and microbial consequences. While rodent models are a useful method for determining causal relationships between HMO, gut microbiota, and behavior, murine studies of gut microbiota usually employ oral gavage, a technique stressful to the mouse. Our aim was to develop a less-invasive technique for $\mathrm{HMO}$ administration to remove the potential confound of gavage stress. Under the hypothesis that stress affects gut microbiota, particularly $B$. infantis, we predicted the pups receiving a prebiotic solution in a less-invasive manner would have the highest amount of Bifidobacteria in their gut. Methods. This study was designed to test two methods, active and passive, of solution administration to mice and the effects on their gut microbiome. Neonatal C57BL/6J mice housed in a specific-pathogen free facility received increasing doses of fructooligosaccharide (FOS) solution or deionized, distilled water. Gastrointestinal (GI) tracts were collected from five dams, six sires, and 41 pups over four time points. Seven fecal pellets from unhandled pups and two pellets from unhandled dams were also collected. Qualitative real-time polymerase chain reaction (qRT-PCR) was used to quantify and compare the amount of Bifidobacterium, Bacteroides, Bacteroidetes, and Firmicutes. Results. Our results demonstrate a significant difference between the amount of 
Firmicutes in pups receiving water passively and those receiving FOS actively ( $p$ value $=0.009$ ). Additionally, we found significant differences between the fecal microbiota from handled and non-handled mouse pups. Discussion. From our results, we conclude even handling pups for experimental purposes, without gavage, may induce enough stress to alter the murine gut microbiota profile. We suggest further studies to examine potential stress effects on gut microbiota caused by experimental techniques. Stress from experimental techniques may need to be accounted for in future gut microbiota studies. 
1 Handling Stress may Confound Murine Gut Microbiota Studies

2 Cary R. Allen-Blevins ${ }^{1}$, Xiaomeng You ${ }^{2}$, Katie Hinde ${ }^{3,4}$, and David A. Sela $2,5,6$

$3 \quad{ }^{1}$ Department of Human Evolutionary Biology, Harvard University, Cambridge MA

$4 \quad{ }^{2}$ Department of Food Science, University of Massachusetts, Amherst MA

$5 \quad{ }^{3}$ Center for Evolution and Medicine, Arizona State University, Tempe AZ

6 4School of Human Evolution and Social Change, Arizona State University, Tempe AZ

7 5Department of Microbiology, University of Massachusetts, Amherst MA

$8{ }^{6}$ Center for Microbiome Research, University of Massachusetts Medical School, Worcester, MA

\section{ABSTRACT:}

Background. Accumulating evidence indicates interactions between human milk composition, particularly sugars (human milk oligosaccharides or HMO), the gut microbiota of human infants, and behavioral effects. Some HMO secreted in human milk is unable to be endogenously digested by the human infant but are able to be metabolized by certain species of gut microbiota, including Bifidobacterium longum subsp. infantis (B. infantis), a species sensitive to host stress (Bailey \&Coe 2004). Exposure to gut bacteria like B. infantis during critical neurodevelopment windows in early life appears to have behavioral consequences; however, environmental, physical, and social stress during this period can also have behavioral and microbial consequences. While rodent models are a useful method for determining causal relationships between $\mathrm{HMO}$, gut microbiota, and behavior, murine studies of gut microbiota usually employ oral gavage, a technique stressful to the mouse. Our aim was to develop a lessinvasive technique for $\mathrm{HMO}$ administration to remove the potential confound of gavage stress. Under the hypothesis that stress affects gut microbiota, particularly $B$. infantis, we predicted the pups receiving a prebiotic solution in a less-invasive manner would have the highest amount of Bifidobacteria in their gut.

Methods. This study was designed to test two methods, active and passive, of solution administration to mice and the effects on their gut microbiome. Neonatal C57BL/6J mice housed in a specific-pathogen free facility received increasing doses of fructooligosaccharide (FOS) solution or deionized, distilled water. Gastrointestinal (GI) tracts were collected from five dams, 


2

six sires, and 41 pups over four time points. Seven fecal pellets from unhandled pups and two pellets from unhandled dams were also collected. Qualitative real-time polymerase chain reaction (qRT-PCR) was used to quantify and compare the amount of Bifidobacterium, Bacteroides, Bacteroidetes, and Firmicutes.

Results. Our results demonstrate a significant difference between the amount of Firmicutes in pups receiving water passively and those receiving FOS actively ( $p$-value=0.009). Additionally, we found significant differences between the fecal microbiota from handled and non-handled mouse pups.

Discussion. From our results, we conclude even handling pups for experimental purposes, without gavage, may induce enough stress to alter the murine gut microbiota profile. We suggest further studies to examine potential stress effects on gut microbiota caused by experimental techniques. Stress from experimental techniques may need to be accounted for in future gut microbiota studies.

\section{INTRODUCTION:}

The gut microbiota has major physiological and potentially biopsychological implications for human health (Lyte 2010, Walter \& Ley 2011, Oh et al. 2010, Allen-Blevins, Sela \& Hinde 2015).

The collection of hundreds of bacterial species in the human gut exerts strong influences on immune function, nutrition, and neurodevelopment through maintaining gut barrier function, fermenting dietary fiber to short-chain fatty acids, and producing neurotransmitters (Dinan et al. 2015, Grenham et al. 2011). Processes such as programming the immune system likely begin with microbial exposure at birth and perturbations in the gut microbiota early in development have been implicated in chronic disease, including psychological conditions (Backhed et al. 2015; Douglas-Escobar, Elliott \& Neu2013; Rook and Lowry 2013). 
54 Recent research in mice suggests early gut microbiota affects neurodevelopment and behavior

55 (Borre et al. 2014, Sudo et al. 2004, Diaz Heijtz et al. 2011). Mice reared without microbiota

56 ("germ-free") exhibit increased corticosterone response to restraint stress and reduced

57 expression levels of brain-derived neurotrophic factor in the hippocampus (Sudo et al. 2004).

58 The increased corticosterone response is partially reversed with exposure to Bifidobacterium

59 longum subsp. infantis, a species dominating the human infant gut (Sudo et al. 2004, Yatsuenko

60 et al.). Notably, colonization with $B$. infantis only partly normalizes the corticosterone response

61 in 6 week old mice, but not 14 week old mice (Sudo et al. 2004). Slightly contrary to this

62 research, germ-free mice showed reduced anxiety behavior in light-dark and elevated maze

63 plus tests (Diaz Heijtz et al. 2011). However, the differences in the type of stressor (restraint

64 stress vs. an open field) may cause these contradictory stress responses in germ-free animals.

65 Regardless, Diaz Heijtz and colleagues also demonstrated only early life colonization of germfree mice, not colonization in mature mice, could normalize the behavior of germ-free mice (Diaz

67 Heijtz et al. 2011). These studies suggest critical neurodevelopmental windows exist in early life during which gut microbiota are crucial to shaping behavior (Borre et al. 2014, Allen-Blevins,

69 Sela \& Hinde 2015).

Evolutionary Context

If early life gut microbiota are critical for normal neurodevelopment, mothers necessarily play an essential role in programming neurodevelopment through transmitting and supporting the microbiota (Backhed et al. 2015, Allen-Blevins, Sela \& Hindeet al. 2015, Sela and Mills 2010). Initial microbial colonization is vertically transmitted from mother to offspring during delivery (Backhed et al. 2015, Dominguez-Bello et al. 2010, Mueller et al. 2015; Hinde \& Lewis 2015). The newly colonized infant gut is then exposed to mother's milk, which in humans contains glycans such as human milk oligosaccharides (HMO) that are not digested by the infant (Marcobal \& Sonnenburg 2012, Sela \& Mills 2010). While the infant does not possess 
80

81

82

endogenous enzymes to cleave $\mathrm{HMO}$, certain species of gut microbiota can metabolize $\mathrm{HMO}$, including B. infantis (Sela \&Mills 2010, Sela et al. 2008, Sela et al. 2011, Sela et al. 2012). B. infantis is capable of metabolizing $\mathrm{HMO}$ as a sole carbon source, and $B$. infantis,

Bifidobacterium longum and Bifidobacterium breve affect stress and anxiety behaviors (Yatsuenko et al. 2012, Sudo et al. 2004, Savignac et al. 2014, Sela \&Mills 2010, Sela et al. 2008). Particular strains of Bifidobacterium can also produce $y$-aminobutyric acid (GABA), a major inhibitory neurotransmitter (Barrett et al. 2012, Yunes et al. 2016). The resulting interactions create a milk-microbiota-brain-behavior (M2B2) system, which may allow mothers to influence infant behavior through their milk (Allen-Blevins, Sela \& Hinde 2015).

\section{Experimental Rationale}

Studying the M2B2 system in model organisms presents unique challenges because experimental techniques can induce stress in animals that affects microbiota and behavior (Hoggatt et al. 2010, Bailey \&Coe 1999, Bailey, Lubach \& Coe. 2004). Stress is a challenge to homeostasis which may be caused by environmental, physiological, social, or psychological stimuli (Bailey 2014, Mendoza in press). Early life stress, including neonatal handling and maternal separation, can have long-term developmental consequences and disrupt the regulation of crucial biopsychological pathways, such as the hypothalamus-pituitary-adrenal axis (Dalmaz et al. 2015). Gut microbiome experiments frequently involve oral gavage of rodents with known bacterial strains, fecal matter, or other compounds (Turnbaugh et al. 2006, Fujimura et al. 2014, Ji et al. 2012.). This technique induces stress responses and can be injurious or fatal to mice, particularly when they are very young (Hoggatt et al. 2010, Flamm 2013). Alterations in gut microbiota in response to host stress have been demonstrated in mice and rhesus macaques (Tarr et al. 2015, Bailey, Lubach \& Coe 2004, Bailey \&Coe 1999). If gut microbiota are sensitive to stress, invasive techniques, like gavage, introduce a potential confound. Changes in microbial profiles over the course of an experiment could be due to the 
106

107

108

109

110

111

112

113

114

115

116

117

118

119

120

121

122

123

124

125

126

127

128

129

130

131

treatment or stress induced from experimental techniques. Since the M2B2 system must be studied prior to weaning, methods such as dosing water or chow with $\mathrm{HMO}$ are not effective. Therefore, a non-invasive technique for administering prebiotic solutions directly to very young mice is necessary.

The purpose of this experiment was to determine a less stressful method for administering experimental prebiotic liquids to conventional mouse pups. Our aims were to develop a method of studying particular diet-microbe interactions in non-gnotobiotic mice. Reducing psychological perturbation was a main goal because bifidobacteria that dominate the infant gut microbiome are reduced after stress exposure (De Loez et al. 2014, Bailey, Lubach \& Coe 2004). Therefore, we predicted pups receiving a prebiotic solution in a more passive manner would have higher amounts of Bifidobacteria. We tested two methods, active and passive, of administering fructooligosaccharide (FOS), a previously demonstrated bifidogenic prebiotic (Howard et al. 1995), to mouse pups from post-natal day 1 to post-natal day 21 (PND1-PND21). FOS was used in this pilot experiment due to its bifidogenic properties and the prohibitive cost of HMO. Bifidobacterium and Bacteroides counts were analyzed because of their potential roles in neurodevelopment (Allen-Blevins, Sela \& Hinde 2015, Hsiao et al. 2013, O’Sullivan et al. 2011), while Bacteroidetes and Firmicutes were analyzed due to these phyla being dominant within human gut microbiomes.

\section{MATERIALS \& METHODS:}

Subjects: We conducted our methodological study (Figure 1) in captive-bred laboratory mice (Mus musculus). Six timed-pregnant C57BL/6J females and six C57BL/6J males were purchased from The Jackson Laboratory (Bar Harbor, Maine, USA) at six weeks old. Animals were housed in the Harvard University Biological Research Infrastructure, a specific-pathogen free facility, under standard Institutional Animal Care and Usage Committee (IACUC) murine 
132 environmental conditions. Water and PicoLab commercial chow were available ad libitum. In

133 consideration of the greater risk of reduced maternal care and increased pup mortality among

134 C57BL/6J primiparae (Brown et al. 1999), initial litters were culled and dams placed in single

135 pair mating cages for one week. These mating pairs produced the litters used for the

136 experimental manipulations. One dam may have still been nulliparous, as she exhibited signs of

137 pregnancy but no litter was observed prior to being placed in a mating cage. However, the litter

138 may have been delivered and cannibalized prior to observation. This would be consistent with

139 other first litters from these dams being cannibalized or found dead. Due to the unexpected

140 death of one male, M1 was mated to F1 and then to F6. The other matings were as follows: M2-

141 F2, M3-F4, M4-F3, and M5-F5. Animals were maintained in breeding cages for seven days

142 before females were removed to individual cages for experimental manipulations. Males

143 remained in single, separate home cages, undisturbed except for cage changes, until

144 euthanasia approximately one week after the birth of their sired litter. All cages were clear

145 plastic, 10.5 inches by 6.5 inches by 5.0 inches.

146

147 Experimental Manipulations: Dams were randomly assigned to the following experimental groups: passive water, passive fructooligosaccharide (FOS), active water, active FOS, buccal water, and buccal FOS. The litters for each group were reduced to six on PND0, with the exception of the active water litter, which only contained five pups at birth. The pregnancy of the buccal water dam failed, leaving no experimental litter for the condition.

Pups in each experimental group were handled daily. At the beginning of each provision of the assigned treatment, the home cage of the litter was placed next to a clean cage with fresh bedding. The dam was removed from the home cage and placed into the clean cage for the duration of pup manipulation for that day. Pups were immobilized by grasping the skin at the nape and along the spine, and rotating their bodies to reveal the ventrum. Starting at PND10, 
158 pups were lifted by the tails prior to grasping the nape. For active FOS and water conditions, a

159 micropipette tip was placed in the pup's mouth and the dosage was injected directly into the oral 160 cavity. In passive conditions, a micropipette tip was used to transfer the dosage to a Crematocrit 161 tube that was then placed near the pup's mouth with the intent to induce the suckling response 162 (Szczypka et al. 1999). As pups in the passive litters aged and the dosages increased (Figure 163 2), only micropipette tips were used to place the dosage near the pups' mouths. The switch to 164 only micropipette tips occurred on PND12 for the passive water group and PND15 for the 165 passive FOS group. All tips and tubes were autoclaved prior to use. For buccal conditions, the 166 daily dosage was micropipetted onto a sterile cotton swab, which was then inserted into the 167 pup's mouth. After receiving the daily dosage, the pup was returned directly to the home cage and the next pup was removed for dosing. Once pups began to open their eyes, they were placed into the clean cage with the dam after dosing. When all pups had received their daily treatment, the dam and pups were returned to the home cage.

FOS was purchased from Sigma-Aldrich Corp. and administered in a 2.5mM solution for PND1PND7and a 25mM solution for PND8-PND21. 25mM was the concentration of $\mathrm{HMO}$ producing results when given to mice from birth to weaning in Kurakevich et al. (2013). The 2.5mM dosage was used to determine whether the $25 \mathrm{mM}$ could be further reduced, to decrease future $\mathrm{HMO}$ cost. Distilled, deionized water was used to create the FOS solutions. Distilled, deionized water was also used for the water conditions.

Sample Collection: Gastrointestinal tracts $(\mathrm{Gl})$ were collected from experimental groups on PND0, PND7, PND14, and PND21, while control fecal samples from non-handled, non-dosed mice were collected on PND14 and PND20. On PND0 as many pups as necessary to reduce litter size to six were anesthetized with carbon dioxide, decapitated with sharp scissors, and their GI tracts were collected. Since the active water litter had five pups at birth, only one pup 
184 was euthanized on PND0. On PND7, one pup from each litter was euthanized in the same 185 manner and their GI tract collected. At PND14, one pup from each litter was euthanized with 186 carbon dioxide and their GI tract collected. On PND21, all remaining pups in the litter were 187 euthanized with carbon dioxide and their GI tracts collected. Dams were also euthanized with 188 carbon dioxide on PND21 and GI tracts were collected. Sires were euthanized with carbon 189 dioxide and GI tracts collected approximately one week after the birth of their litter. GI tracts

190 were snap-frozen in dry ice and stored at -80C until analysis. Voided fecal pellet samples were 191 also collected on PND14 and PND20 from non-handled C57BL/6J pups and two non-handled 192 dams housed in the same facility, matched for living conditions and diet, which served as controls to the treatment groups. Animal use was approved by the Harvard University Institutional Animal Care and Use Committee under protocol 14-08-217.

qRT-PCR Analysis:

197 Fecal pellet and GI tract material was transferred from Harvard University, Cambridge,

198

199

200

201

202

203

204

205

206

207

208
Massachusetts on dry ice to University of Massachusetts-Amherst, Amherst, Massachusetts for quantitative real-time polymerase chain reaction (qRT-PCR) analysis. GI tracts were thawed on ice and fecal material from the tracts was scraped into sterile sample tubes. Samples were unattainable from PND0 GI tracts $(\mathrm{N}=9)$, due to lack of fecal matter within the tracts.

Additionally, samples from the buccal FOS group ( $F 6, N=10)$ were not analyzed because there was no litter from F3 (the buccal water dam).

DNA was extracted using a bead beating protocol (FastPrep-24 ${ }^{\mathrm{TM}} 5 \mathrm{G}$ MP Biomedicals Inc, US) and standard protocol for the QIAmp DNA stool kit (Qiagen, Valencia, CA, US). DNA quality was determined via nanodrop (Thermo Fisher Scientific, Waltham, MA, US) and extracted DNA was diluted to $4 \mathrm{ng} / \mu \mathrm{L}$. Custom TaqMan gene expression assays (Thermo Fisher Scientific) for 
209

210

211

212

213

214

215

216

217

218

219

220

221

222

223

224

225

226

227

228

229

230

231

232

233 234

Bifidobacterium, Firmicutes, Bacteroides, and Bacteroidetes were designed using the following sequences:

Bifidobacterium (Penders et al. 2005):

Forward primer: GCGTGCTTAACACATGCAAGTC

Reverse primer: CACCCGTTTCCAGGAGCTATT

Probe: TCACGCATTACTCACCCGTTCGCC

Firmicutes (Lecerf et al. 2012):

Forward primer: GAATCTTCCACAATGGACGAAAG

Reverse primer: AATACCGTCAATACCTGAACAGTTACTC

Probe: CTGATGGAGCAACGCCGCGT

Bacteroides (Layton et al. 2006):

Forward primer: GAGAGGAAGGTCCCCCAC

Reverse primer: CGCTACTTGGCTGGTTCAG

Probe: CCATTGACCAATATTCCTCACTGCTGCCT

Bacteroidetes (Dick \& Field 2004):

Forward primer: AACGCTAGCTACAGGCTTAACA

Reverse primer: ACGCTACTTGGCTGGTTCA

Probe: CAATATTCCTCACTGCTGCCTCCCGTA

Samples were run in triplicate, with negative control blanks of RNAse free water and a standard curve included on each 96-well plate, using Applied Biosystems 7500 Fast Real-Time PCR system (Thermo Fisher Scientific). Wells included $1 \mu \mathrm{L}$ of TaqMan gene expression assay, $10 \mu \mathrm{L}$ of TaqMan master mix (Thermo Fisher Scientific), $5 \mu \mathrm{L}$ of RNAse free water, and $4 \mu \mathrm{L}$ of the DNA sample for a total of $20 \mu \mathrm{L}$ in each well. Plates were run at $50 \mathrm{C}$ for 2 minutes, $95 \mathrm{C}$ for 10 minutes, and then 45 cycles of $95 \mathrm{C}$ for 15 seconds and $60 \mathrm{C}$ for 1 minute. 
235

236

237

238

239

240

241

242

243

244

245

246

247

248

249

250

251

252

253

254

255

256

257

258

259

260

Statistical Analysis:

The Kruskal-Wallis test was performed in RStudio (Version 0.98.1103) to compare variation in

Bifidobacterium, Firmicutes, Bacteroides, and Bacteroidetes quantity between treatment groups:

active fructooligosaccharide (FOS), passive FOS, active water, and passive water. Wilcoxon

rank sum tests were performed in RStudio (Version 0.98.1103) to compare variation in

Bifidobacterium, Bacteroides, and Bacteroidetes quantity in the combined totality of the

treatment groups (active FOS, passive FOS, active water, and passive water) versus the fecal

pellet samples from non-handled mice housed in the same facility, for both pup and dam

samples. Since the sample sizes were very unequal for non-handled control $(N=7)$ versus

treatment $(\mathrm{N}=21)$ pups, Wilcoxon tests were also performed to compare the quantity of

Bifidobacterium, Bacteroides, and Bacteroidetes in the combined treatment groups against the fecal pellet samples from pups on PND14 and PND21. For PND14, sample sizes were equal at four control and four treatment pups. As no fecal pellet samples were collected on PND21 and no GI tracts were collected on PND20, the fecal pellet samples from PND20 were compared to

GI tract samples from PND21. Significance for the Kruskal-Wallis and Wilcoxon tests was established as $p<0.05$. One sample, the FOS passive PND7, was excluded from analysis due to bacterial detection levels below the standard curve.

\section{RESULTS:}

\section{Gastrointestinal (GI) Tract Samples}

$\log 10$ colony-forming unit (CFU) equivalents/ng of DNA were measured for Bifidobacterium, Bacteroides, Bacteroidetes, and Firmicutes for each pup treatment group ( $\mathrm{N}=21$, Figure 3). All treatment group samples demonstrated some amount of Bacteroidetes, Bacteroides, and Firmicutes. Maximum, minimum, and median counts, as well as the interquartile range, for each taxa are listed in Table 1. One data point was excluded from the Bifidobacterium counts due to a lack of agreement among triplicate samples (active water, PND7). From a total of 20 
261 treatment samples, qRT-PCR revealed the majority (19) to have $<10^{1}$ quantity of bifidobacteria.

262 The Kruskal-Wallis test determined no significant difference in the median bacterial counts

263 across treatment groups for Bacteroidetes $(p=0.546)$, Bacteroides $(p=0.534)$, or Bifidobacterium

$264(p=0.786)$. However, there was a significant difference for Firmicutes $(p=0.043)$.

265

266 To determine which treatment groups were significantly different in the amount of Firmicutes,

267 Wilcoxon rank sum tests were performed in a pairwise fashion. There were no significant

268 differences in Firmicutes amounts between the passive water and active water groups

$269(p=0.171)$, passive water and passive FOS groups $(p=0.247)$, active water and active FOS

270 groups $(p=0.067)$, or active FOS and passive FOS groups $(p=0.329)$. However, there was a

271 significant difference between the median Firmicutes counts for the passive water $\left(10^{3}\right)$ and

272 active FOS groups $\left(10^{4} ; p=0.009\right)$.

273

274 Bacterial counts were also quantified for samples from the $\mathrm{Gl}$ tracts of the litter sires $(\mathrm{N}=4$,

275 Figure 4) and the dams of each treatment group and the buccal water dam with no litter ( $N=5$,

276 Figure 5). Maximum, minimum and median counts, along with the interquartile range, are in

277 Table 2. The maximum Bifidobacterium count for the sires came from the sire mated to the

278 buccal water dam, which produced no litter. All samples for the treatment dams contained $<10^{1}$

279 Bifidobacterium.

280

281 Fecal Pellet Samples

282 Fecal pellet samples from non-handled pups and dams were measured for Bifidobacterium,

283 Bacteroides, Bacteroidetes, and Firmicutes log10 CFU equivalents/ng of DNA as a control

284 (Figure 3 for pups, Figure 5 for dams). Maximum, minimum, and median counts, with the

285 interquartile ranges, can be found in Table 1 for the pups, while the same counts for the dams 
286

287 288 289

can be found in Table 2. The maximum Bifidobacterium count of $10^{3}$ was found in two pup samples. For the dams, both samples contained $10^{1}$ Bifidobacterium.

\section{Gastrointestinal Tract (GI) versus Fecal Pellet Samples}

Since there were no significant differences in bacterial counts of Bacteroidetes, Bacteroides, or Bifidobacterium across treatment groups, we combined these groups for analysis against the fecal pellet controls. The Wilcoxon rank sum test revealed significant differences between counts for all bacterial taxa (Bifidobacterium $p$ value $<0.001$, Bacteroidetes $p$ value $=0.008$, Bacteroides $\mathrm{p}$ value $<0.001$ ) in the treatment groups and bacterial counts for the fecal pellets collected from pups (Figure 3).

Comparisons by post-natal day also revealed significant differences between non-handled and handled pups. Wilcoxon rank sum tests demonstrated significant differences in the medians of the control and treatment samples for Bacteroides (control median: $10^{4}\left(I Q R=<10^{1}\right)$, treatment median: $10^{3}\left(I Q R=10^{2}\right)$, $p$-value $\left.=0.03\right)$ and Bifidobacterium (control median: $10^{2}\left(I Q R=<10^{1}\right)$, treatment median: $<10^{1}\left(I Q R=<10^{1}, p\right.$-value=0.03) on PND14, but an non-significant difference for Bacteroidetes ( $p$-value=0.057, Figure 6). These significant differences for Bacteroides ( $p$ value $=0.003)$ and Bifidobacterium $(p$-value $=0.006)$ median counts in the control and treatment samples were also evident at PND21, despite a large difference in sample size (control N=3, treatment $\mathrm{N}=14$ ).

Wilcoxon rank sum tests found no significant difference between samples from the two control dams and samples from the treatment dams $(\mathrm{N}=4)$. This lack of significance was maintained when the F3 dam, who had no litter and received no treatment, was included (Figure 5).

\section{DISCUSSION:}


312 In this experiment, we specifically focused on Bifidobacterium, Firmicutes, Bacteroides and

313 Bacteroidetes, phyla frequently studied in animal models due to their presence and

314 hypothesized importance in the human gut. While quantifying and studying the gut microbiota as

315 a whole is a necessary step to fully understand interactions between the host and microbiota,

316 we narrowed our focus to these phyla because of our particular aims for this study.

317 Bifidobacterium and Bacteroides are both good candidate genera for containing species that

318 potentially affect neurodevelopment during infancy. Both genera have previously been shown to

319 contain species, such as Bifidobacterium longum subsp. infantis and Bacteroides fragilis, that

320 can affect behavior, possibly through neurodevelopmental pathways (Allen-Blevins, Sela \&

321 Hinde 2015, Sudo et al. 2004, Hsiao et al. 2013). Firmicutes and Bacteroidetes were also

322 quantified due to their correlation with obesity, inverse correlation with each other, and frequent

323 measurement in gavage experiments (Turnbaugh et al. 2006, Li et al. 2015).

324

325

326

327

328

329

330

331

332

333

334

335

336

337

338

The major finding of this paper is the significant decrease in Bifidobacterium Bacteroides, and

Bacteroidetes present in the gut microbiota of handled animals provided either water or FOS.

Cognizant of stress effects on bifidobacteria (Bailey, Lubach \& Coe 2004), our experiment was designed to determine a less invasive technique to administer bifidogenic compounds to neonatal mice. While the ultimate goal was to create a method for administration of human milk oligosaccharides (HMO) to mice, FOS was used in this initial experiment due to the prohibitive cost of HMO. We expected to find the most bifidobacteria in the group passively fed FOS, since this was expected to be the least stressful technique and included supplementation of a compound previously demonstrated as bifidogenic (Howard et al. 1995). However, there were no significant differences in Bacteroidetes, Bacteroides, or Bifidobacterium across our treatment groups, falsifying our prediction. There was a significant difference between the median amount of Firmicutes in the pups provided water passively and the pups actively provided with FOS.

These two treatment groups were different on both factors (treatment method and substance), 
339

340

341

342

343

344

345

346

347

348

349

350

351

352

353

354

355

356

357

358

359

360

361

362

363

364

so the significant difference is not suprising. Additionally, administration of FOS is correlated with an increase in Firmicutes (Li et al. 2015). The median amounts of Firmicutes across treatment groups remained between $10^{3}$ and $10^{4}$, while the control pups had a median amount of $10^{6}$. While we were unable to statistically compare these values due to the difference in treatment groups for Firmicutes, there seems to be a substantial difference between the amount of Firmicutes in the control and treatment groups. The statistically significant differences in quantities of Bacteroides, Bacteroides, and Bifidobacterium between the control and treatment groups suggest a factor common to all four treatment groups may have affected these phyla of gut microbiota.

Lack of initial exposure to Bifidobacterium, Bacteroides, and Bacteroidetes and differences between voided fecal pellets and GI tract fecal samples may explain the differences in bacterial quantities between our handled and non-handled animals; however we think these are unlikely explanations. The absence of bifidobacteria in the handled dams creates the possibility of the handled pups lacking bifidobacteria because they had no exposure. However, the counts of Bifidobacterium in the water sires indicate at least the dams for the water groups were exposed to bifidobacteria for seven days. Sires were cohoused with the dams for one week and thecoprophagic habit of mice (Heinrichs 2001) makes dam exposure to bifidobacteria highly likely. While both the FOS sires and dams appear to be lacking bifidobacteria, one of the FOS pups had the highest count of bifidobacteria in the treatment groups $\left(>10^{1}\right)$ Therefore, nonexposure to bifidobacteria is unlikely to explain our results. Non-exposure also cannot explain the differences in Bacteroides and Bacteroidetes, as all of the treated dams contained these taxa. Additionally, the differences in the use of voided fecal pellet samples from the control animals (collected from cages) and internal fecal samples from the treatment animals (collected from the distal colon of $\mathrm{GI}$ tracts) are also unlikely to explain the magnitude of difference in bifidobacteria. Fecal samples in both mice and humans have demonstrated much higher 
365

366

367

368

369

370

371

372

373

374

375

376

377

378

379

380

381

382

bifidobacterial counts than gut lumen samples (Marcotte and Lavoie 1996, Ouwehand et al. 2004). However, our treatment samples were of intact feces collected from the distal colon and not samples of the mucosa or luminal fluid. Also, the two water sires had counts of Bifidobacterium in stool from their intestinal tract that were greater than the counts in the voided fecal pellets of the control dams. Therefore, while some variation may be expected due to differences in sample collection, it is unlikely to reach the magnitude of differences between the median bifidobacteria from the control pups and the treatment pups.

While non-exposure to bacteria is an unlikely explanation, the stress of handling, common to all treatment groups, may have contributed to the changes across the treatment group gut microbiota. Bifidobacterium, known to be susceptible to host stress (Bailey, Lubach \& Coe 2004), was decreased to the point of $>10^{1}$ in the majority of our treated mice, the largest decrease of any of the bacterial taxa in our study. Since all of the control animals and two of our minimally handled sires largely maintained Bifidobacterium in their gut, and all animals were of the same genetic background, in the same facility on the same diet, the sharp decrease in this taxa known to be stress-sensitive, is likely due to a stressor. It should be noted that this explanation may not be valid for the lack of Bifidobacterium in the FOS sires. Additionally, Lactobacillus, a genus within the Firmicutes phylum is also stress-sensitive (Bailey 2014). While the significant difference in Firmicutes between the passive water pups and active FOS pups suggests administration of FOS was able to increase the amount of Firmicutes, all of the treatment groups still had a median amount $10^{2}-10^{3}$ below the median amount of the control group. Therefore, handling stress could potentially be driving decreases in the other bacterial taxa as well, to varying degrees. Both passive and active handling techniques appear to negatively influence the amount of Bifidobacterium, Bacteroides, and Bacteroidetes in the guts of our treated subjects, with the quantity of Bifidobacterium being the most severely affected. 
391 If even non-invasive handling stress is potentially correlated with significant changes to the gut

392 microbiota and the loss of an entire taxon, this creates a particular challenge for research

393 centerd on the gut microbiota and early life development. To adequately study a potential milk-

394 microbiota-brain-behavior (M2B2) pathway, supplementation of animal models with HMO may

395 be necessary (Allen-Blevins, Sela \& Hinde 2015). While gavage is frequently used to administer

396 compounds to rodents, this technique can stress the animal (Flamm 2012, Hoggatt et al.

397 2010).Stress in early life, such as maternal separation, is correlated with significant changes gut

398 microbiota (Bailey \& Coe 1999). If non-invasive handling is provoking a stress response in

399 laboratory animals significant enough to affect gut microbiota, then handling young animals

400 during microbiota experiments may confound the results. For example, handling all of the

401 animals, including controls and those receiving vehicles, may lead a researcher to conclude

402 there are none or reduced levels of a bacterium that may actually be diminished due to handling

403 stress. Such a reduction in bacterial taxa may mask potential interactions between an

404 experimental compound and bacterial taxa that might be present if the compound was

405 administered without stress. More research is necessary to determine if common experimental

406 techniques are creating confounds in murine microbial studies.

407

408

Synbiotics, a combination of prebiotics and the bacteria of interest (Schrezenmeir \& de Vrese

409

2001), and communal use of non-handled control animals may mitigate the potential challenges

410

of handling stress affecting gut microbiota. Since synbiotics provide both the substrate for

411

bacterial growth and the bacteria (Schrezenmeir \&de Vrese 2001), they may provide a method

412 for studying the most stress sensitive bacteria. For both human and animal studies, exposing

413 the subject to the desired bacteria daily may continually replace the diminished strains and

414 mimic the effects of permanent colonization for the duration of exposure. Microbial and other

415 changes can then be compared in these animals to non-handled control animals. As a guiding

416 principle of the American Association for Laboratory Animal Science (Committee for the Update 
417 of the Guide for the Care and Use of Laboratory Animals, 2011) is understandably to reduce the

418 number of animals used in experiments, there may be a reluctance to include negative control

419 animals that are not handled at all. The reduction principle can still be achieved for gut

420 microbiota studies if multiple labs share feces and potentially other data from non-handled

421 animals housed in the same facilities, on the same diets, and from the same genetic

422 backgrounds.

423

424 Our study has several caveats and limitations. It is important to note the small sample sizes in

425 our study. The small number of control samples compared to the treatment samples may have

426 impacted our results. To determine if handling is truly causing such a large difference in gut

427 microbiota, replicating this study with a greater number of control samples would be of great

428 value. We also did not take corticosterone measurements of the mice, which would have

429 allowed us to quantify their physiological stress reaction to handling. Additionally, the stress of

430 handling does not explain the lack of bifidobacteria in the FOS sires or the buccal water dam.

431 Since this dam had no litter, she was also minimally handled. However, these animals contained

432 counts of Bacteroidetes, Bacteroides, and Firmicutes on par with their sex and age-matched

433 conspecifics. After removal from mating cages, all dams were housed separately until

434 parturition. While isolation of pregnant females to prevent cannibalism of pups is an accepted

435 practice (Committee for the Update of the Guide for the Care and Use of Laboratory Animals,

436 2011), since this female never had a litter, the social stress of isolation may have affected her

437 differently. The FOS sires may have lacked bifidobacteria due to an unnoticed illness or a

438 difference in genotype (Bevins \& Salzman 2011; Wacklin et al. 2011), but this is simply

439 speculation. Therefore, since these two animals present a conundrum and it is highly important

440 to determine the extent of handling effects on gut microbiota, this research should be repeated

441 with larger sample sizes and including measures of corticosterone. Prior to that, researchers

442 should remain cognizant of potential handling effects on their data. 
444 Additionally, we used quantitative real-time PCR (qRT-PCR) to quantify the bacteria number in

445 our samples, which is regarded as a sensitive and specific method to detect commensal

446 bacteria (Castillo et al. 2006). Though qRT-PCR is regarded as an accurate method, we found

447 some samples to contain more Bacteroides than Bacteroidetes which might be due to qRT-PCR

448 amplification bias. Thus, primers should be carefully designed to ensure the same amplification

449 efficiency among the bacteria of interest in future studies.

450

451

CONCLUSION:

Although more research is clearly necessary, the stress of handling, or even the social stress of isolation, may have the capacity to affect murine gut microbiota. Particularly when using young animals to investigate microbial responses to prebiotics, such as studies focusing on the potential milk-microbiota-brain-behavior (M2B2) system, care should be taken during experiments to ensure necessary controls and accurate data collection. Sharing fecal samples from control animals of the same genetic background, housed in the same facility, and fed the same diet would met standards of animal reduction, while enabling comparison of handled treatment animals to non-handled animals.

ACKNOWLEDGEMENTS: The authors thank Prof. Rachel Carmody for providing the fecal pellet control samples and Harvard University's Institute for Qualitative Social Science for statistical guidance. Our gratitude to the three anonymous reviewers for their comments that improved the manuscript.

\section{REFERENCES:}

Allen-Blevins CR, Sela DA, Hinde K. 2015. Milk bioactives may manipulate microbes to mediate parent-offspring conflict. Evol Med Public Health doi:10.1093/emph/eov007

Bäckhed F, Roswall J, Peng Y, Feng Q, Jia H. 2015. Dynamics and stabilization of the human gut microbiome during the first year of life. Cell Host and Microbe 
472

473

474

475

476

477

478

479

480

481

482

483

484

485

486

487

488

489

490

491

492

493

494

495

496

497

498

499

500

501

502

503

504

505

506

507

508

509

510

511

512

513

514

515

516

517

518

519

520

521
Bailey MT. 2014. Influence of stressor-induced nervous system activation on the intestinal microbiota and the importance for immunomodulation. In: Lyte M, Cryan JF (eds). Microbial Endocrinology: The Microbiota-Gut-Brain Axis in Health and Disease. New York: Springer, 2014,255-76. doi: 10.1007/978-1-4939-0897-4_12.

Bailey MT, Coe CL. 1999. Maternal separation disrupts the integrity of the intestinal microflora in infant rhesus monkeys. Dev Psychobiol 146-155.

Bailey MT, Lubach GR, Coe CL. 2004. Prenatal stress alters bacterial colonization of the gut in infant monkeys. J Ped Gastroenterol Nut 38:414-421.

Bevins CL, Salzman NH. 2011. The potter's wheel: the host's role in sculpting its microbiota. Cellular and molecular life sciences : CMLS 68:3675-85.

Brown RE, Mathieson WB, Stapleton J, Neumann PE. 1999. Maternal behavior in female C57BL/6J and DBA/2J inbred mice. Physiology and Behavior 67:599-605

Castillo M. Martín-Orúe SM, Manzanilla EG, Badiola I, Martín M, Gasa J. 2006. Quantification of total bacteria, enterobacteria and lactobacilli populations in pig digesta by real-time PCR. Vet Microbiol, 114:165-70.

Christian LM, Galley JD, Hade EM, Schoppe-Sullivan S, Kamp-Dush C, Bailey MT. 2014. Gut microbiome composition is associated with temperament during early childhood. Brain, Behavior, and Immunity

Committee for the Update of the Guide for the Care and Use of Laboratory Animals, 2011. Guide for the Care and Use of Laboratory Animals. 8th ed. National Academies Research Press. Washington DC.

Dalmaz C, Noschang C, Krolow R, Raineki C, Lucion AB. 2015. How postnatal insults may program development: Studies in animal models. In: Antonelli MC (ed). Perinatal Programming of Neurodevelopment. New York: Springer, 2015,121-147

Dave M, Higgins P, Middha S, Rioux KP. 2012. The human gut microbiome: current knowledge, challenges, and future directions. Trans Res 160:246-257.

de Leoz MLA, Kalanetra KM, Bokulich NA, Strum JS, Underwood MA, German JB, Mills DA, Lebrilla CB. 2014. Human milk glycomics and gut microbial genomics in infant feces show a correlation between human milk oligosaccharides and gut microbiota: a proof-ofconcept study. J Proteome Res

Desbonnet, Garrett, Clarke, Kiely, Cryan. 2010. Effects of the probiotic Bifidobacterium infantis in the maternal separation model of depression.

Diaz Heijtz R, Wang S, Anuar F, Qian Y, Björkholm B, Samuelsson A, Hibberd ML, Forssberg $H$, Pettersson S. 2011. Normal gut microbiota modulates brain development and behavior. Proceedings of the National Academy of Sciences of the United States of America 108:3047-52.

Dick LK, Field KG. 2004. Rapid estimation of numbers of fecal Bacteroidetes by use of a quantitative PCR assay for 16S rRNA genes. Appl Environ Microbiol. 70:5695-5697.

Dinan TG, Stilling RM, Stanton C, Cryan JF. 2015. Collective unconscious: How gut microbes shape human behavior. J Psych Res 1-9

Douglas-Escobar M, Elliott E, Neu J. 2013. Effect of intestinal microbial ecology on the developing brain. JAMA pediatrics 167:374-9.

Flamm E. 2012. Neonatal animal testing paradigms and their suitability for testing infant formula. Toxicology Mechanisms and Methods 23:57-67.

Fujimura KE, Demoor T, Rauch M, Faruqi AA, Jang S, Johnson CC, Boushey HA, Zoratti E, Ownby D, Lukacs NW, Lynch SV. 2014. House dust exposure mediates gut microbiome Lactobacillus enrichment and airway immune defense against allergens and virus infection. Proceedings of National Academy of Sciences 111:805-810.

Grenham S, Clarke G, Cryan JF, Dinan TG. 2011. Brain-gut-microbe communication in health and disease. Front Physiol 2:94. 
522

Heinrichs, SC. 2001. Mouse feeding behavior: ethology, regulatory mechanisms and utility for mutant phenotyping. Behavioral Brain Research 125:81-88.

Hinde, K., \& Lewis, Z. T. (2015). Mother's littlest helpers. Science, 348(6242), 1427-1428.

Hsiao E, McBride S, Hsien S, Sharon G, Hyde E, McCue T, Codelli J, Chow J, Reisman S, Petrosino J, Patterson P, Mazmanian S. 2013. Microbiota modulate behavioral and physiological abnormalities associated with neurodevelopmental disorders. Cell 155:1451-63.

Hoggatt AF, Hoggatt J, Honerlaw M, Pelus LM. 2010. A spoonful of sugar helps the medicine go down: a novel technique to improve oral gavage in mice. Journal of the American Association for Laboratory Animal Science 49:329-334.

Howard MD, Gordon DT, Garleb KA, Kerley MS. 1995. Dietary fructooligosaccharide, xylooligosaccharide and gum arabic have variable effects on cecal and colonic microbiota and epithelial cell proliferation in mice and rats. J Nutr 2604-2609.

Ji YS, Kim HN, Park HJ, Lee JE, Yeo SF, Yang JS, Park SY, Yoon HS, Cho GS, Franz CMAP, Bomba A, Shin HK, Holzapfel WH. 2012. Modulation of the murine microbiome with a concomitant anti-obesity effect by Lactobacillus rhamnosus GG and Lactobacillus sakei NR28. Beneficial Microbes 3:13-22.

Kurakevich E, Hennet T, Hausmann M, Rogler G, Borsig L. 2013. Milk oligosaccharide sialyl $(\alpha 2,3)$ lactose activates intestinal CD11c+ cells through TLR4. Proceedings of the National Academy of Sciences of the United States of America 110:17444-9.

Layton A, McKay L, Williams D, Garrett V, Gentry R, Sayler G. 2006. Development of Bacteroides 16S rRNA gene TaqMan-based real-time PCR assays for estimation of total, human, and bovine fecal pollution in water. Appl Environ Microbiol 72:4214-4224.

Lecerf JM, Dépeint F, Clerc E, Dugenet Y, Niamba CN, Rhazi L, Cayzeele A, Abdelnour G, Jaruga A, Younes H, Jacobs H, Lambrey G, Abdelnour AM, Pouillart PR. 2012. Xylooligosaccharide (XOS) in combination with inulin modulates both the intestinal environment and immune status in healthy subjects, while XOS alone only shows prebiotic properties. Br J Nutr 108:1847-1858.

Li S, Yingyi G, Chen L, Lijuan G, Ou S, Peng X. 2015. Lean rats gained more body weight from a high-fructooligosaccharide diet. Food Funct. 6:2315.

Limmathurotsakul D, Golding N, Dance DAB, Messina JP, Pigott DM, Moyes CL, Rolim DB, Bertherat E, Day NPJ, Peacock SJ, Hay SL. 2016. Predicted global distribution of Burkholderia pseudomallei and burden of melioidosis. Nature Microbiol 1:15008.

Lyte M. 2010. The microbial organ in the gut as a driver of homeostasis and disease. Med Hypo 74: 634-638.

Marcobal A, Sonnenburg JL. 2012. Human milk oligosaccharide consumption by intestinal microbiota. Clin Microbiol Infect 18:12-15.

Mendoza SP. Social Stress: Concepts, assumptions, and animal models. In: Hormones, Brain, and Behavior. Elsevier; Oxford. In press.

Mueller N, Bakacs E, Combellick J, Grigoryan Z, Dominguez-Bello M. 2014. The infant microbiome development: mom matters. Trends in molecular medicine.

Oh PL, Benson AK, Peterson DA, Patil PB, Moriyama EN, Roos S, Walter J. 2010. Diversification of the gut symbiont Lactobacillus reuteri as a result of host-driven evolution. ISME J 4:377-387.

O'Mahony SM, Marchesi JR, Scully P, Codling C. 2009. Early life stress alters behavior, immunity, and microbiota in rats: implications for irritable bowel syndrome and psychiatric illnesses. Biological Psychiatry

O'Sullivan E, Barrett E, Grenham S, Fitzgerald P, Stanton C, Ross RP, Quigley EMM, Cryan JF, Dinan TG. 2011. BDNF expression in the hippocampus of maternally separated rats: does Bifidobacterium breve 6330 alter BDNF levels? Beneficial Microbes 2:199-207. 
572

Penders J, Vink C, Driessen C, London N, Thijs C, Stobberingh EE. 2005. Quantification of Bifidobacterium spp., Escherichia coli and Clostridium difficile in faecal samples of breast-fed and formula-fed infants by real-time PCR. FEMS Microbiol Lett 243:141-147. Rook GAW, Lowry CA, Raison CL. 2013. Microbial 'Old Friends', immunoregulation and stress resilience. Evolution, Medicine, and Public Health 46-64.

Salter SJ, Cox MJ, Turek EM, Calus ST, Cookson WO, Moffatt MF, Turner P, Parkhill J, Loman NJ, Walker AW. 2014. Reagent and laboratory contamination can critically impact sequence-based microbiome analyses. BMC Biol 12:87.

Sela DS, Chapman J, Adeuya A, Kim JH, Chen F, Whitehead TR, Lapidus A, Rokshar DS, Lebrilla CB, German JB, Price NP, Richardson PM, Mills DA. 2008. The genome sequence of Bifidobacterium longum subsp. infantis reveals adaptations for milk utilization within the infant microbiome. Proceedings of the National Academy of Sciences. 48:18964-18969.

Sela DA, Garrido D, Lerno L, Wu S, Tan K, Eom H, Joachimiak A, Lebrilla C, Mills DA. 2012. Bifidobacterium longum subsp. infantis ATCC $15697 \alpha$-fucosidases are active of fucosylated human milk oligosaccharides. Applied and Environmental Microbiology 78:795-803.

Sela DA, Li Y, Lerno L, Wu S, Marcobal AM, German JB, Chen X, Lebrilla CB, Mills DA. 2011. An infant-associated bacterial commensal utilizes breast milk sialyloligosaccharides. Journal of Biological Chemistry 286:11909-11918.

Sela DA and Mills DA. 2010. Nursing our microbiota: molecular linkages between bifidobacteria and milk oligosaccharides. Trends Microbiol 18:298-307.

Schrezenmeir J and de Vrese M. 2001. Probiotics, prebiotics, and synbiotics-approaching a definition. Am J Clin Nutr 73(2):361s-364s.

Smith MI, Yatsunenko T, Manary MJ, Trehan I, Mkakosya R, Cheng J, Kau AL, Rich SS, Concannon P, Mychaleckyj JC, Liu J, Houpt E, Li JV, Holmes E, Nicholson J, Knights D, Ursell LK, Knight R, Gordon JI. 2013. Gut microbiomes of Malawian twin pairs discordant for kwashiorkor. Science Transl Med 339:548-554.

Sudo N, Chida Y, Aiba Y, Sonoda J, Oyama N, Yu X-N, Kubo C, Koga Y. 2004. Postnatal microbial colonization programs the hypothalamic-pituitary-adrenal system for stress response in mice. J Physiol. 558: 263-275.

Szczypka MS, Rainey MA, Kim DS, Alaynick WA, Marck BT, Matsumoto AM, Palmiter RD. 1999. Feeding behavior in dopamine-deficient mice. Proc Natl Acad Sci 96:1213812143.

Turnbaugh PJ, Ley RE, Mahowald MA, Magrini V, Mardis ER, Gordon JI. 2006. An obesityassociated gut microbiome with increased capacity for energy harvest. Nature 444:10271031.

Turnbaugh PJ, Ridaura VK, Faith JJ, Rey FE, Knight R, Gordon JI. 2009. The effect of diet on the humanized gut microbiome: A metagenomics analysis in humanized gnotobiotic mice. Science Transl Med 1:6ra14.

Walter J, Ley R. 2011. The human gut microbiome: Ecology and recent evolutionary changes. Ann Rev Microbiol 65:411-429.

Wacklin P, Makivuokko H, Alakulppi N, Nikkila J, Tenkanen H, Rabina J, Partanen J, Aranko K, Matto, J. 2011. Secretor genotype (FUT2 gene) is strongly associated with the composition of Bifidobacteria in the human intestine. PLoS One. DOI: 10.1371/journal.pone.0020113

Yatsunenko T, Rey FE, Manary MJ, Trehan I, Dominguez-Bello MG, Contreras M, Magris M, Hildago, G, Baldassano RN, Anokhin AP, Heath AC, Warner B, Reeder J, Kuczynski J, Caporaso JG, Lozupone CA, Lauber C, Clemente JC, Knights D, Knight R, Gordon JI. 2012. Human gut microbiome viewed across age and geography. Nature. 486: 222-228. 
622 Yunes RA, Poluektova EU, Dyachkova MS, Klimina KM, Kovtun AS, Averina OV, Orlova VS, 


\section{Timeline for Treatment Groups}

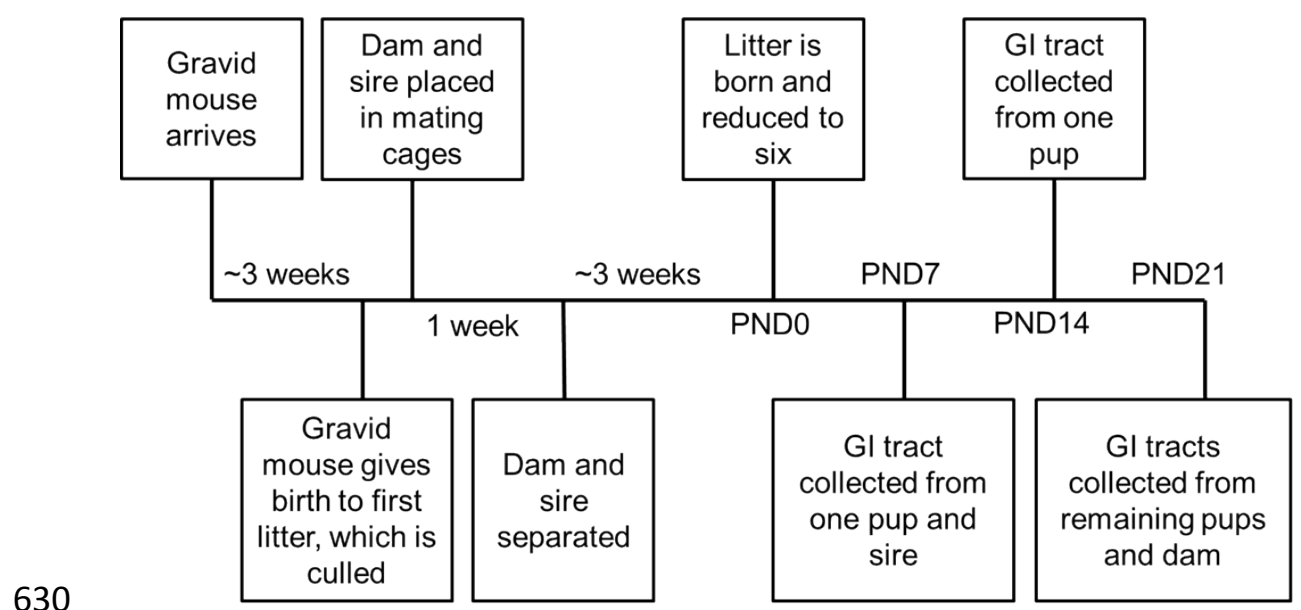

Figure 1. Timeline for each treatment group. Samples were collected at time points postnatal day (PND)0, PND7, PND14, and PND21. 


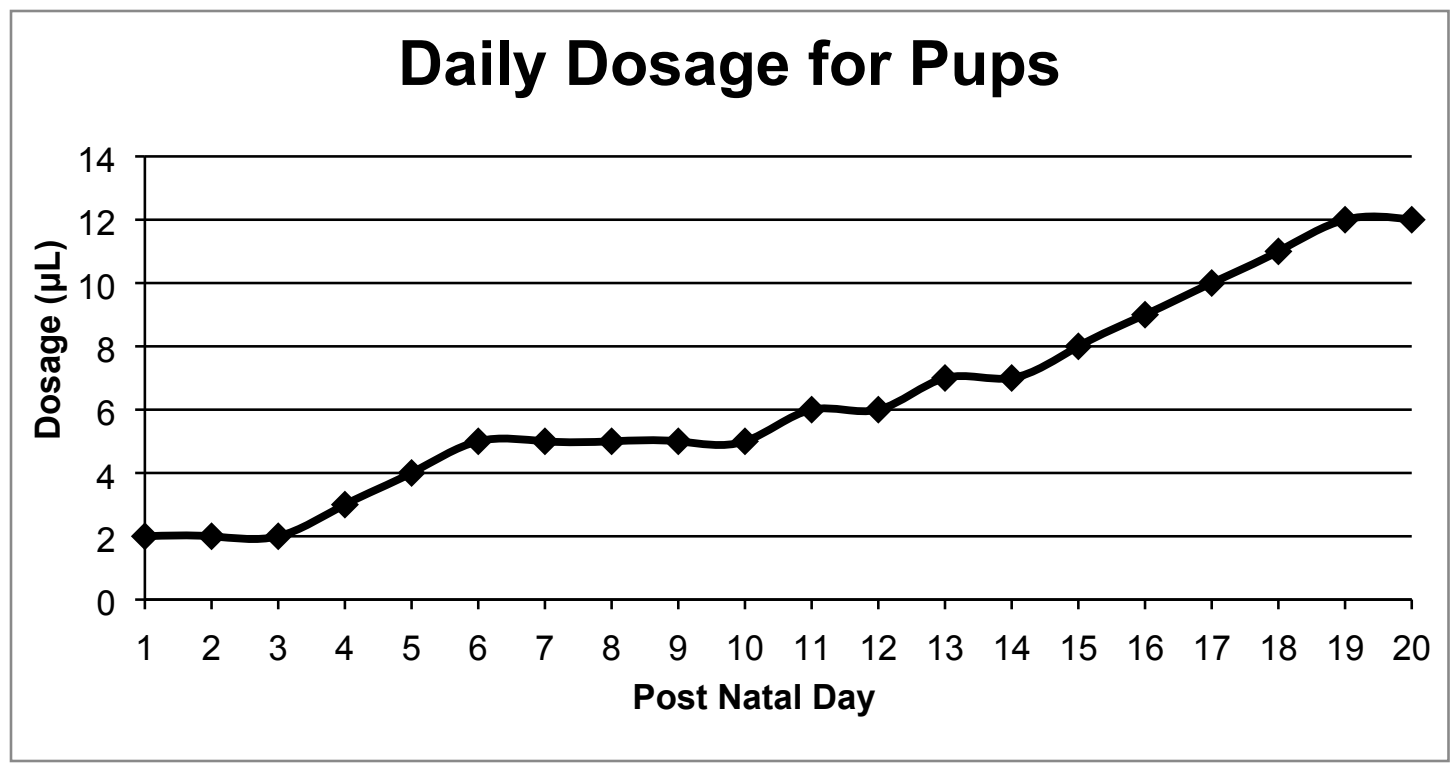

Figure 2. Daily dosage of FOS or water in microliters.

652

653

654

655

656

657

658

659

660

661

662

663

664

665

666

667

668

669

670

671

672

673

674

675

676

677

678

679

680

681

682

683 


\section{log10 CFU Equivalents for Control and Treatment Groups}

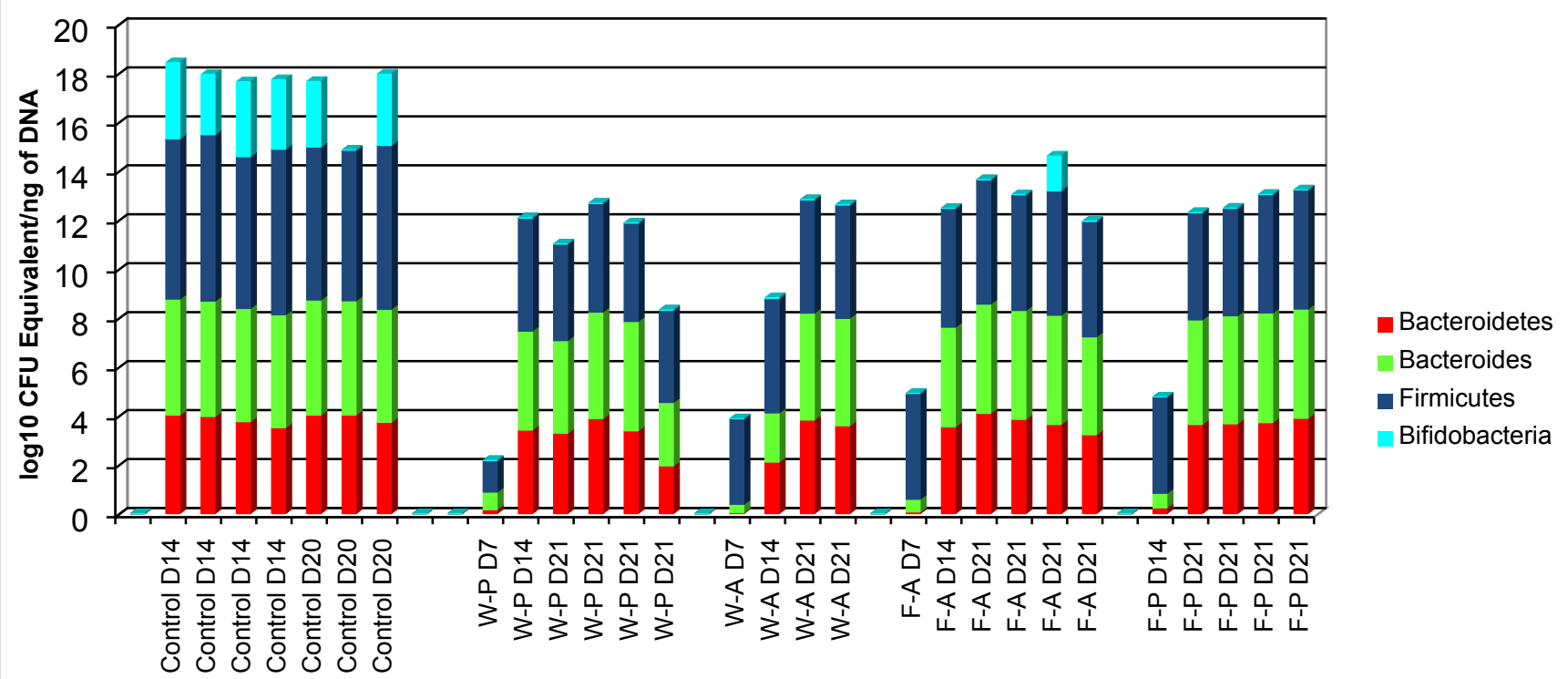

\section{Group and Day of Collection}

Figure 3. log10 Colony-forming unit (CFU) equivalents/ng of sample DNA for control and treatment groups. Wilcoxon rank sum test demonstrated significant differences between the control and treatment samples for all Bifidobacterium $(p<0.001)$, Bacteroides $(p<0.001)$, and Bacteroidetes ( $p$-value $=0.008$ ). There was also a significant difference for Firmicutes between the WP and FA groups ( $p$-value=0.009). $W-P=$ water passive, $W-A=$ water active, $F-A=F O S$ active, $\mathrm{F}-\mathrm{P}=\mathrm{FOS}$ passive. 
Figure 4. log10 Colony-forming unit (CFU) equivalents/ng of sample DNA for feces collected from the $\mathrm{GI}$ tracts of treatment litter sires.

\section{log10 CFU Equivalents for Sires}

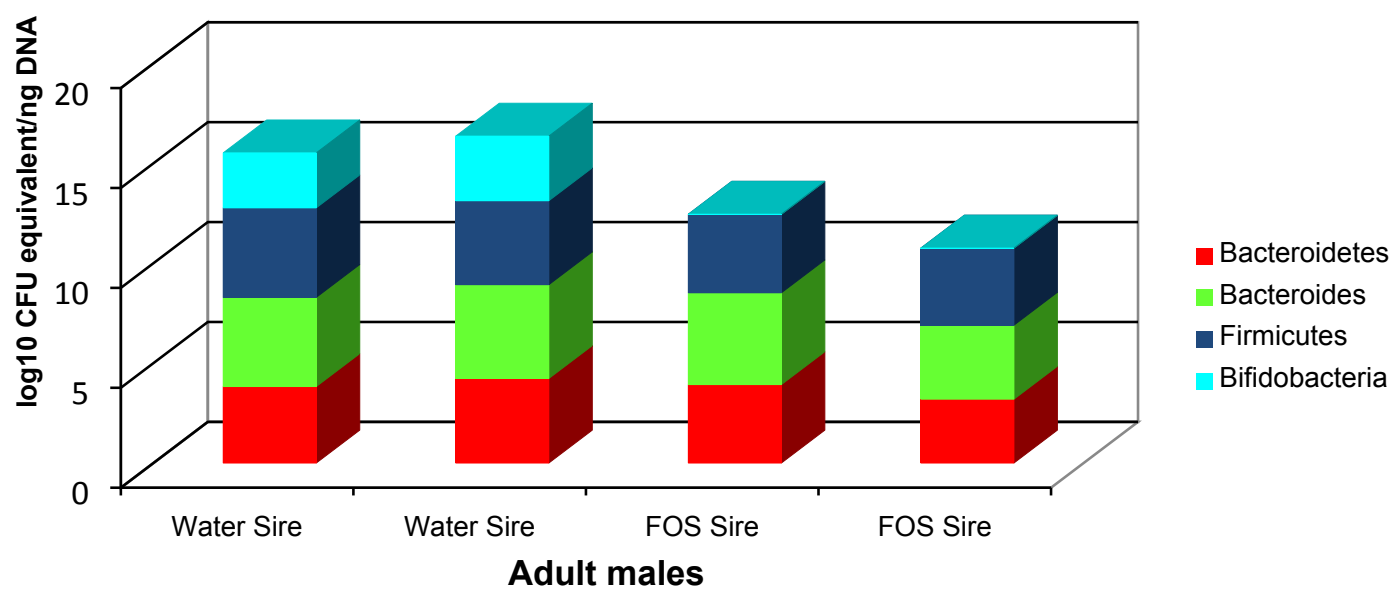

705

706

707

708

709

710

711

712

713

714

715

716 


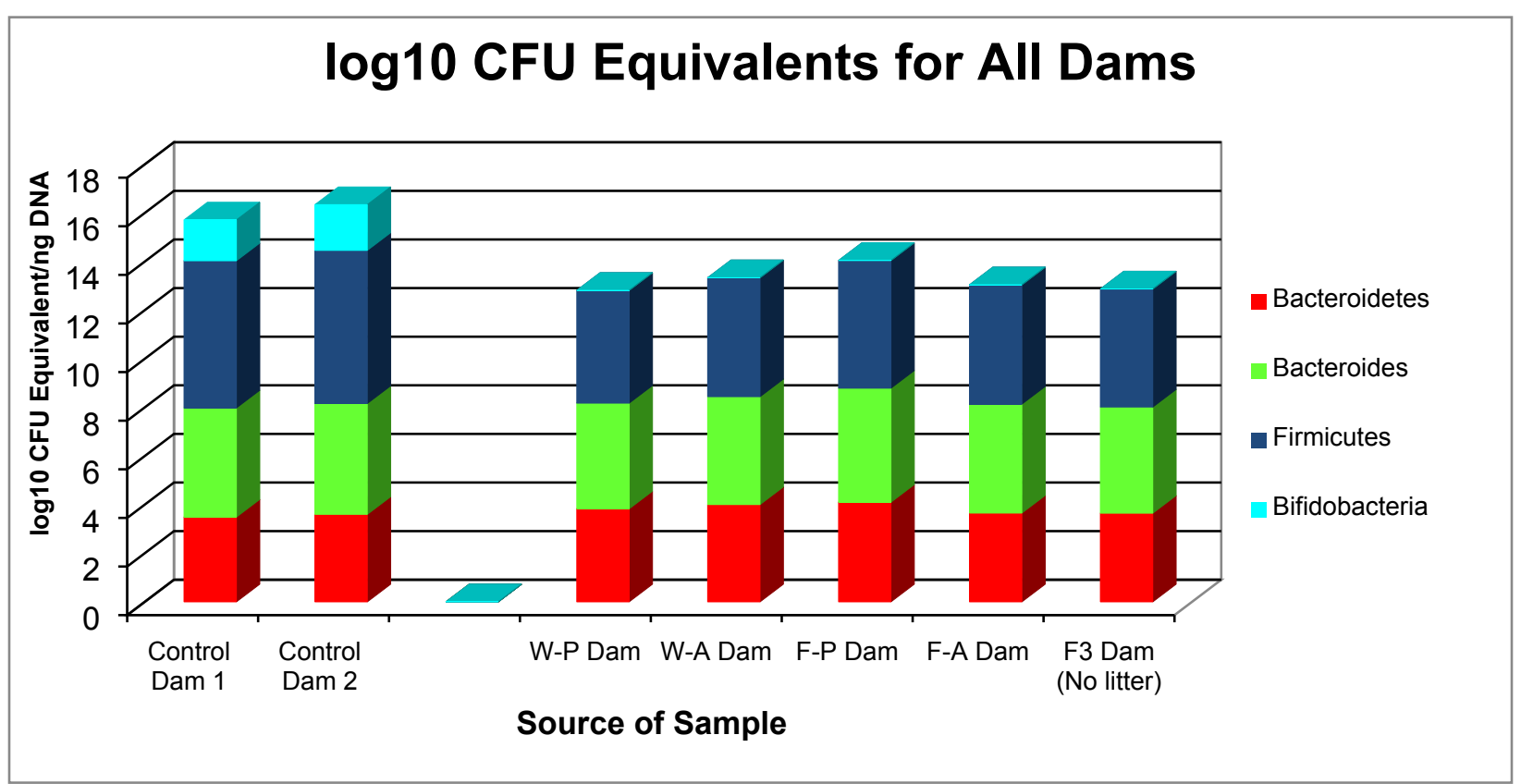

Figure 5. log10 Colony-forming units (CFU) equivalents/ng of sample DNA for control and experimental dams. There was no significant difference between the samples, despite the treatment dams having no bifidobacteria. The F3 dam was the buccal water dam that did not deliver a litter. $\mathrm{W}-\mathrm{P}=$ passive water, $\mathrm{W}-\mathrm{A}=$ active water, $\mathrm{F}-\mathrm{A}=$ active $\mathrm{FOS}, \mathrm{F}-\mathrm{P}=$ passive $\mathrm{FOS}$. 


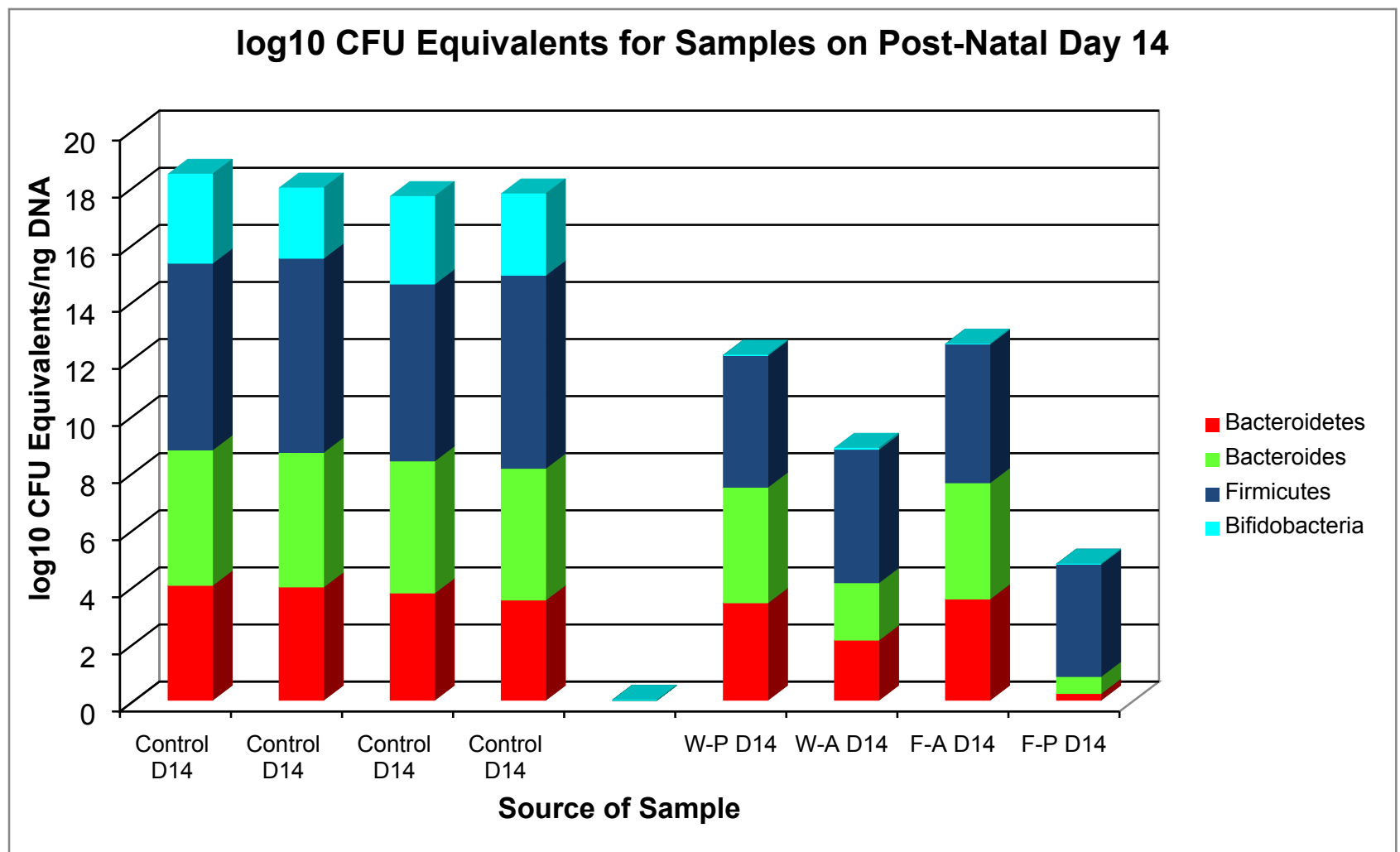

Figure 6. log10 Colony-forming unit (CFU) equivalents/ng of sample DNA from control and treatment samples collected on post-natal day 14. The median Bifidobacterium and Bacteroides counts in the control samples were significantly different from the treatment samples ( $p$-value $=0.03$ for both comparisons) as tested by Wilcoxon rank sum test. The medians of Bacteroidetes counts were not significantly different between the treatment and control groups ( $p$-value $=0.057$ ). Firmicutes could be compared between control and treatment groups, because there were significant differences between treatment groups for this taxa. W$\mathrm{P}=$ passive water, $\mathrm{W}-\mathrm{A}=$ active water, $\mathrm{F}-\mathrm{A}=$ active $\mathrm{FOS}, \mathrm{F}-\mathrm{P}=$ passive $\mathrm{FOS}$. 


\begin{tabular}{|c|c|c|c|c|}
\hline Group/Taxa & Maximum & Minimum & Median & $\begin{array}{l}\text { Interquartile } \\
\text { Range }\end{array}$ \\
\hline $\begin{array}{l}\text { GI Tract-Pups } \\
(\mathrm{N}=21) \\
\text { Bacteroidetes }\end{array}$ & $10^{4}$ & $<10^{1}$ & $10^{3}$ & $10^{1}$ \\
\hline Bacteroides & $10^{4}$ & $<10^{1}$ & $10^{4}$ & $10^{1}$ \\
\hline Bifidobacterium & $10^{1}$ & $<10^{1}$ & $<10^{1}$ & $<10^{1}$ \\
\hline Firmicutes & $10^{5}$ & $10^{1}$ & $10^{4}$ & $<10^{1}$ \\
\hline $\begin{array}{l}\text { Fecal Pellets- } \\
\text { Pups }(\mathrm{N}=7) \\
\text { Bacteroidetes }\end{array}$ & $10^{4}$ & $10^{3}$ & $10^{3}$ & $<10^{1}$ \\
\hline Bacteroides & $10^{4}$ & $10^{4}$ & $10^{4}$ & $<10^{1}$ \\
\hline Bifidobacterium & $10^{3}$ & $<10^{1}$ & $10^{2}$ & $<10^{1}$ \\
\hline Firmicutes & $10^{6}$ & $10^{6}$ & $10^{6}$ & $<10^{1}$ \\
\hline
\end{tabular}
samples. Maximum, minimum, median, and interquartile range (IQR)counts for Bacteroidetes, Bacteroides, Bifidobacterium, and Firmicutes in treatment ( $\mathrm{Gl}$ tract, $\mathrm{N}=21$ ) and control (Fecal pellets, $\mathrm{N}=7$ ) pups. 


\begin{tabular}{|c|c|c|c|c|}
\hline Group/Taxa & Maximum & Minimum & Median & $\begin{array}{l}\text { Interquartile } \\
\text { Range }\end{array}$ \\
\hline $\begin{array}{l}\text { GI Tract-Dams }(\mathbf{N}=5) \\
\text { Bacteroidetes }\end{array}$ & $10^{4}$ & $10^{3}$ & $10^{3}$ & $<10^{1}$ \\
\hline Bacteroides & $10^{4}$ & $10^{4}$ & $10^{4}$ & $<10^{1}$ \\
\hline Bifidobacterium & $<10^{1}$ & $<10^{1}$ & $<10^{1}$ & $<10^{1}$ \\
\hline Firmicutes & $10^{5}$ & $10^{4}$ & $10^{4}$ & $<10^{1}$ \\
\hline $\begin{array}{l}\text { GI Tract-Sires }(\mathbf{N}=\mathbf{4}) \\
\text { Bacteroidetes }\end{array}$ & $10^{4}$ & $10^{3}$ & $10^{3}$ & $<10^{1}$ \\
\hline Bacteroides & $10^{4}$ & $10^{3}$ & $10^{4}$ & $<10^{1}$ \\
\hline Bifidobacterium & $10^{3}$ & $<10^{1}$ & $10^{1}$ & $10^{2}$ \\
\hline Firmicutes & $10^{4}$ & $10^{3}$ & $10^{4}$ & $<10^{1}$ \\
\hline $\begin{array}{l}\text { Fecal Pellets-Dams }(\mathrm{N}=\mathbf{2}) \\
\text { Bacteroidetes }\end{array}$ & $10^{3}$ & $10^{3}$ & $10^{3}$ & $<10^{1}$ \\
\hline Bacteroides & $10^{4}$ & $10^{4}$ & $10^{4}$ & $<10^{1}$ \\
\hline Bifidobacterium & $10^{1}$ & $10^{1}$ & $10^{1}$ & $<10^{1}$ \\
\hline Firmicutes & $10^{6}$ & $10^{6}$ & $10^{6}$ & $<10^{1}$ \\
\hline
\end{tabular}

Table 2. log 10 Colony-forming unit (CFU) equivalents/ng of sample DNA for dams and 